\title{
Knowledge and mindset on anaphylaxis management in children and teenagers among caregivers in Slovenia
}

\author{
Eva Šoster Križnik ${ }^{1 *}$, Ljubica Deisinger ${ }^{2}$, Mirjana Maslar ${ }^{1}$, Andreja Obermayer ${ }^{1}$, Vesel Tina $^{3}$ \\ From Food Allergy and Anaphylaxis Meeting 2014 \\ Dublin, Ireland. 9-11 October 2014
}

\section{Background}

Appropriate first-line management of anaphylaxis is based on its recognition and application of intramuscular adrenaline as soon as possible. Parents, other caregivers including kindergarten and school personnel and also children themselves are first-line actors in case of anaphylaxis in a child or teenager and therefore comprehensive and thorough training for them is essential.

\section{Methods}

We have applied ten short questions to parents and other caregivers (including kindergarten and school personnel) considering ability of their anaphylaxis recognition and management and willingness to help in case of anaphylaxis. The same questionnaire was used before and after 2-hour long course on recognition and management of anaphylaxis in three allergological centers in Slovenia.

\section{Results}

138 participants were included. $75 \%$ of participants recognised correctly signs of anaphylaxis before and $74 \%$ after the course. Regarding the correct position during anaphylaxis there were $77 \%$ correct answers before the course and $99 \%$ after. The rate of correct answers about adrenaline being the most important drug during the anaphylaxis increased from $72 \%$ before the course to $100 \%$ afterwards. Regarding the correct order of management during the anaphylaxis there were $57 \%$ correct answers in the pre-course questionnaire and $94 \%$ in the post-course questionnaire. $33 \%$ of participants answered correctly the question about the correct application of

${ }^{1}$ Children's Department, General Hospital Celje, Celje, Slovenia

Full list of author information is available at the end of the article adrenaline auto-injector Epipen before the course and $93 \%$ after the course.

$70 \%$ of participants would give adrenaline by auto-injector to the child with anaphylaxis before the course and $96 \%$ after the course. $29 \%$ of all participants were worried about the side effects of adrenaline, 34\% of all about the legal consequences and $20 \%$ about the injury of a child with a needle. $43 \%$ of the participants believed public awareness campaign would contribute to better management of anaphylaxis in children, $34 \%$ of all thought the regulation of legislation is needed. Only $13 \%$ felt competent to help the child with anaphylaxis before the course but after the course that percentage raised to $71 \%$.

\section{Conclusions}

The 2-hour courses on anaphylaxis management enhanced theoretical ability and the willingness of appropriate first-line management of anaphylaxis in caregivers. Recognition of symptoms and signs of anaphylaxis was more difficult to teach. If the adrenaline auto-injector is prescribed to a child or teenager such trainings should be provided.

\section{Authors' details \\ ${ }^{1}$ Children's Department, General Hospital Celje, Celje, Slovenia. ${ }^{2}$ Outpatient Pediatric Clinic for Allergology and Pulmonology, Ankaran, Slovenia. ${ }^{3}$ Department of Allergology, Rheumatology and Clinical Immunology, University Children's Hospital, University Medical Center, Ljubljana, Slovenia.}

Published: 30 March 2015

doi:10.1186/2045-7022-5-S3-O4

Cite this article as: Šoster Križnik et al:: Knowledge and mindset on anaphylaxis management in children and teenagers among caregivers in Slovenia. Clinical and Translational Allergy 2015 5(Suppl 3):O4. 